\title{
Mendelian susceptibility to mycobacterial disease in tuberculosis-hyperendemic South Africa
}

\author{
H M Cornelissen, ${ }^{1} \mathrm{MB}$ ChB, BSc (Diet); B Glanzmann, ${ }^{2,3} \mathrm{PhD}$; A van Coller, ${ }^{4} \mathrm{MSc}$; C Engelbrecht, ${ }^{2} \mathrm{MSc}$; \\ D R Abraham, ${ }^{5}$ MB ChB, FC Paed (SA), MMed (Paed), Cert Paed Rheumatol (SA); K Reddy, ${ }^{6}$ MB ChB, FC Path (SA), MMed (Micro); \\ M Möller, ${ }^{2,3} \mathrm{PhD}$; C Kinnear, ${ }^{2,3} \mathrm{PhD}$; R H Glashoff, ${ }^{4} \mathrm{PhD}$; M Esser, ${ }^{4,5} \mathrm{MB}$ ChB, FC Paed (SA), MMed (Paed), Cert Paed Rheumatol (SA)
${ }^{1}$ Division of Haematopathology, National Health Laboratory Service and Faculty of Medicine and Health Sciences, Stellenbosch University and Tygerberg Hospital, Cape Town, South Africa
${ }^{2}$ DSI-NRF Centre of Excellence for Biomedical Tuberculosis Research, SAMRC Centre for Tuberculosis Research, Division of Molecular Biology and Human Genetics, Faculty of Medicine and Health Sciences, Stellenbosch University, Cape Town, South Africa
${ }^{3}$ SAMRC Genomics Centre, Cape Town, South Africa
${ }^{4}$ Immunology Unit, Division of Medical Microbiology, National Health Laboratory Service and Faculty of Medicine and Health Sciences, Stellenbosch University and Tygerberg Hospital, Cape Town, South Africa
${ }^{5}$ Department of Paediatrics and Child Health, Faculty of Medicine and Health Sciences, Stellenbosch University and Tygerberg Hospital, Cape Town, South Africa
${ }^{6}$ Division of Medical Microbiology, National Health Laboratory Service and Faculty of Medicine and Health Sciences, Stellenbosch University and Tygerberg Hospital, Cape Town, South Africa

Corresponding author: H M Cornelissen (hcornelissen@sun.ac.za)

\begin{abstract}
Background. Severe infections in the absence of secondary immunodeficiency can alert clinicians to single-gene inborn errors of immunity/ primary immunodeficiency disorders (PIDDs). Mendelian susceptibility to mycobacterial disease (MSMD) is characterised by selective susceptibility to mycobacterial infections due to inborn errors in the interleukin 12-interferon gamma pathway. The South African (SA) burden of hyperendemic tuberculosis (TB) infection provides an interesting context for the study of MSMD.

Objectives. To evaluate whether severe, persistent, unusual or recurrent (SPUR) definitions of TB can be applied in the context of MSMD in SA. Methods. This study is a retrospective review of an SA PIDD cohort. Patients aged 0 - 15 years with SPUR TB infections, assessed between 2013 and 2018, were identified using a proposed algorithm. HIV infection or other secondary causes for immunodeficiency were excluded. Basic investigations, then focused immunophenotyping and next-generation sequencing, were performed.

Results. A total of 20 patients with a clinical diagnosis of MSMD were identified. A further two, forming part of a family cohort, had pathogenic variants but remain asymptomatic. Infection with Mycobacterium tuberculosis complex predominated (64\%), while $27 \%$ had BCG infection or non-tuberculous mycobacteria (NTM) infection. Molecular analysis revealed pathogenic variants in $41 \%$ of patients with SPUR mycobacterial infection, mainly in those with BCG/NTM infection.

Conclusions. In the SA paediatric population, SPUR TB infections, particularly BCG/NTM, in the absence of secondary immunodeficiency, can alert to possible MSMD. The molecular diagnosis is pivotal, guiding disease classification and influencing clinical approach and management. The diagnosis is complex and requires a multidisciplinary approach with close collaboration between clinical immunologists, bioinformaticians, immunologists, clinical geneticists and genetic counsellors.
\end{abstract}

S Afr Med J 2021;111(10):998-1005. https://doi.org/10.7196/SAMJ.2021.v111i10.15341

Immunodeficiency, whether primary or secondary, results in the disruption of normal immune response and function. Typical presentations of immune deficiency are increased susceptibility to infection, usually in a specific class depending on the immune pathway or cell affected..$^{[1,2]}$ The phenotype is broad, and autoimmunity, immune dysregulation and increased risk for malignancy may also be hallmarks of the immunodeficiency. ${ }^{[2,3]}$ In the absence of secondary immunodeficiency, severe infections in otherwise healthy children can alert clinicians to single-gene inborn errors of immunity or primary immunodeficiency disorders (PIDDs)..$^{[1,4]}$

In tuberculosis (TB)-hyperendemic settings such as South Africa (SA), the risk for infection with TB cumulates with exposure, which provides an interesting context for the study of PIDD, in particular Mendelian susceptibility to mycobacterial disease (MSMD). ${ }^{[-8]}$ MSMD is molecularly characterised by errors in the interleukin 12 (IL-12) and interferon gamma (IFN- $\gamma$ ) pathway. ${ }^{[9]}$ IFN- $\gamma$ is a key cytokine in both innate and adaptive responses against viruses and intracellular bacteria, namely mycobacteria. ${ }^{[9-11]}$
There is a dynamic balance between the host and the organism that determines the course of TB infection. ${ }^{[4,12-15]}$ The risk of progression to active disease is highest in infants aged $<12$ months and lowest in children aged 5 - 10 years, the 'wonder years. ${ }^{[6]}$ Severe disease implies that there is either poor host control of the infection, i.e. dissemination, or a complex disease manifestation, often with severe sequelae such as TB empyema or pericarditis. ${ }^{[15]}$ Non-severe disease implies that the host has managed to control the pathogen, resulting in contained disease. ${ }^{[15]}$

Impaired response to IFN- $\gamma$ is caused by mutations in the IFNGR1, IFNGR2, STAT1, CYBB, IRF8 and NEMO genes. ${ }^{[9,10]}$ Deficient production of IFN- $\gamma$ is associated with mutations in $I L 12 B$, IL12RB1, IL12RB2, TYK2, SPPL2a and ISG15. ${ }^{[9,10]}$ MSMD is a PIDD characterised by selective predisposition to infections, typically with BCG or poorly pathogenic mycobacteria (e.g. Mycobacterium avium complex, M. kansasii, M. ghodii). ${ }^{[10,12]}$ Individuals with MSMD are also predisposed to infection with M. tuberculosis complex (MTBC), Salmonella species, Listeria, Candida species, Toxoplasma species, and 
viruses in combination with mycobacteria. ${ }^{[10,12]}$ MSMD is not the only PIDD with increased risk for TB infection; others include severe combined immunodeficiency, common variable immunodeficiency, combined immunodeficiency, chronic granulomatous disease and hyper-IgM syndromes, and must be considered in the differential diagnosis. ${ }^{[13,14]}$

Hyperendemic selective pressure in SA could result in increased exposure of hosts with inborn errors of immunity to a particular infection, revealing their phenotype and resulting in severe, persistent or unusual infection. The study of MSMD in this context may expand the typically described phenotype. ${ }^{[13]}$ The clinical presentation of MSMD is vast, ranging from severe forms of early-onset disseminated, persistent and life-threatening mycobacterial disease to the less severe forms with later-onset mild disease. ${ }^{[1,10,12]}$ The identification of patients with MSMD remains a clinical challenge, particularly in TB-endemic regions such as SA. ${ }^{[13]}$ Clinical definitions and guides to facilitate investigation of patients with possible MSMD in SA are also lacking.

\section{Objectives}

To evaluate the SA phenotype and assess whether severe, persistent, unusual or recurrent (SPUR) definitions of TB can be applied in the context of MSMD in SA, with the aim of providing clinical guides to facilitate identification and diagnosis of such patients.

\section{Methods}

A retrospective review of the SA PIDD registry was done to identify relevant patients for inclusion in this study. Entry onto the SA PIDD registry follows International Union of Immunological Societies (IUIS) and European Society for Immunodeficiencies (ESID) definitions for PIDD, requiring rigorous assessment. ${ }^{[1,2]}$ Patients included in this study were assessed between 2013 and 2018 using the clinical algorithm summarised in Fig. 1, were aged between 0 and 15 years, and had SPUR TB infections in the absence of HIV or other causes of secondary immunodeficiency or anatomical risk. Investigations into a possible diagnosis of MSMD included the use of clinical assessment, basic immune assays, immune phenotyping and next-generation sequencing (NGS).

Baseline investigations included confirmatory HIV enzyme-linked immunoassay (ELISA)/polymerase chain reaction depending on age, full blood count $(\mathrm{FBC})$ and differential count, lymphocyte phenotyping, vaccine antibody responses and a neutrophil burst.

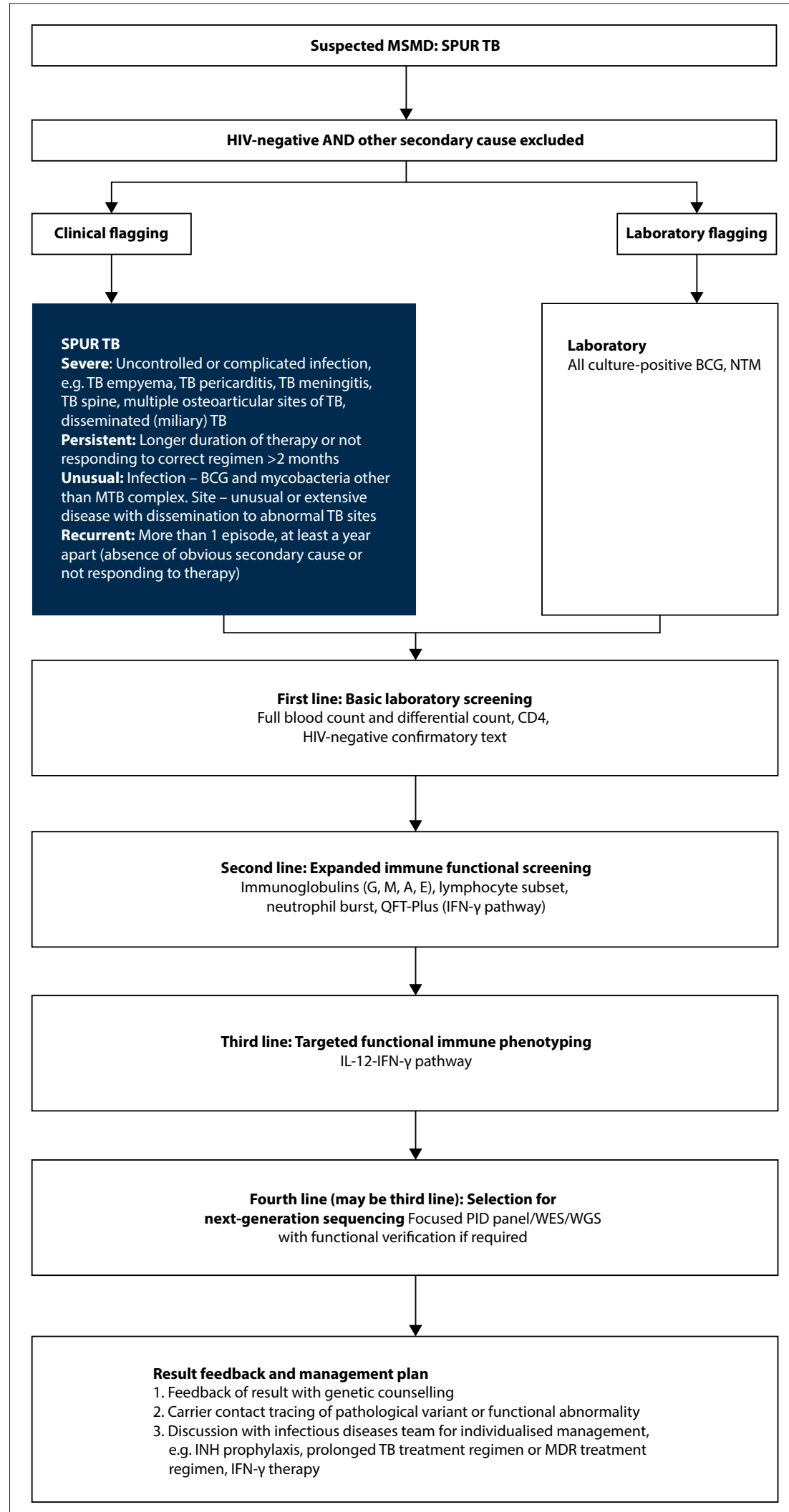

Fig. 1. Algorithm for the identification and management of suspected MSMD. (MSMD = Mendelian susceptibility to mycobacterial disease; SPUR = severe, persistent, unusual or recurrent; $T B=$ tuberculosis; $M T B=$ Mycobacterium tuberculosis; $N T M=$ non-tuberculous mycobacteria; $Q F T$ Plus = QuantiFERON-TB Gold Plus test; IFN- $\gamma=$ interferon gamma; IL-12 = interleukin 12; PID = primary immunodeficiency; WES = whole-exome sequencing; $W G S=$ whole-genome sequencing; $I N H=$ isoniazid MDR = multidrug resistant . 
Definitions for SPUR infections were primarily obtained through expert opinion as well as from definitions of severe TB in children: ${ }^{[5,15]}$

- Severe. Uncontrolled or complicated infection, e.g. TB empyema, TB pericarditis, TB meningitis, TB spine, multiple osteoarticular sites of TB, disseminated (miliary) TB.

- Persistent. Longer duration of therapy or not responding to correct regimen and adherent to therapy $>2$ months intensive phase.

- Unusual. Infection - BCG and non-tuberculous mycobacteria (NTM). Site - unusual or extensive disease with dissemination to abnormal TB sites (ears, brain), severe TB $>1$ site, osteoarticular $\mathrm{TB}$ at an unexpected site (e.g. skull, facial bones).

- Recurrent (5 - 15 years). More than 1 episode, at least 1 year apart (absence of obvious secondary cause or not responding to therapy).

\section{Immunophenotyping}

Where sufficient peripheral blood mononuclear cells (PBMCs) could be isolated from whole blood, phenotyping and functional profiling were performed to assess the integrity of the IL-12IFN- $\gamma$ pathway. Cytokine production was measured using ELISA of supernatants from stimulation experiments, with both IFN- $\gamma$ induced IL-12 production and IL-12-induced IFN- $\gamma$ production being assessed. Where feasible, the QuantiFERON-TB Gold Plus test (QFT-Plus; Qiagen, Germany) was performed using standard laboratory protocols. This test is an IFN- $\gamma$ release assay measuring a functional IFN- $\gamma$ secretory pathway in response to in vitro stimulation by peptide antigens associated with $M$. tuberculosis but not BCG strains and most NTM, with the exception of M. kansaii, M. szulgai and M. marinum.

\section{Molecular diagnosis}

Molecular diagnosis in patients with suspected MSMD was performed using research-based whole-exome sequencing (WES). Initially WES was done via international collaboration, but as of 2013 both WES and immunophenotyping were performed in house. All samples were sequenced on the Ion Proton at the Stellenbosch University Central Analytical Facility using existing protocols. Variants were prioritised using an in-house method, TAPER. Variants identified through WES were validated via Sanger sequencing and classified using American College of Medical Genetics and Genomics and Association for Molecular Pathology guidelines for variant classification.

Results were interpreted through the PIDDGEN group, a multidisciplinary team consisting of a clinical immunologist, clinicians, immunologists, bioinformaticians, molecular geneticists, a clinical geneticist and a genetic counsellor. Relevant genetic results were then returned to the patients through a genetic counsellor. Where applicable, family cascade testing was offered.

\section{Results}

A summary of the clinical, genetic and immunophenotypical information of this cohort of MSMD patients is presented in Table 1.

A total of 22 candidates were entered on the SA PIDD registry with a molecular or clinical diagnosis of MSMD using ESID and IUIS definitions. ${ }^{[1,2]}$ Two of these, forming part of a family cohort of carrier identification, were asymptomatic. The results of baseline laboratory investigations were normal in the majority of patients. Patient 22 was the only patient with a non-responsive neutrophil burst. The majority of the patients presented at $<5$ years of age (59\%), and 59\% were male and $41 \%$ female. Infection with MTBC predominated, in $64 \%$ of patients, while $27 \%$ had BCG or NTM infection. Most (82\%) presented with severe infection, and $50 \%$ with unusual infection. Those with persistent infection (64\%) required a longer duration of treatment to clear disease. Recurrent episodes were seen in $55 \%$, with some patients having $>5$ episodes of TB. Pulmonary presentation predominated (41\%), with central nervous system and gastrointestinal presentation also common.

Functional immunophenotyping of the IL-12-IFN- $\gamma$ pathway was done for 13 patients. All pathogenic variants showed impaired response and no IFN- $\gamma$ autoantibodies. Impaired function was also seen in the $36 \%$ who had either a variant of unknown significance (VUS) or no significant variant found. QFT-Plus was only performed for 6 patients. Patient 22 had a negative QFT result with a suspected BCG infection.

Molecular diagnosis using WES identified possible candidate variants in genes that have previously been associated with MSMD. ${ }^{[9,10]}$ Variants were classified as pathogenic in $41 \%$ of cases. In $9 \%$, no candidate variant was identified and in $50 \%$ a candidate variant classified as a VUS was identified. VUSs require further functional validation and scientific evidence to define their role in disease. The sensitivity of SPUR definitions for identifying a molecular diagnosis of MSMD was $78 \%$. The accuracy of MSMD diagnosis was $35 \%$; however, if a BCG/NTM infection was identified with a SPUR presentation, the accuracy for a pathogenic variant was $57 \%$.

Clinical management was influenced by both pathogenic and VUS identification. Molecular diagnosis allowed haematopoietic stem cell transplantation in patient 21, but unfortunately he died. Pathogenic variant identification in patient 14 allowed close monitoring and early identification and management of her daughter. Carrier testing in patient 22 identified the mother as a carrier, influencing family planning and decisions on sibling testing. Pathogenic variant identification in patient 22 immediately resulted in an extended individualised treatment regimen and closer monitoring. Of particular importance in pathogenic variants was increased close monitoring and surveillance.

\section{Discussion}

Diagnosis of MSMD requires careful clinical evaluation, functional immunophenotyping, and molecular diagnosis where possible. ${ }^{[1,9,10]}$ Often the findings on standard laboratory work-up are normal and not suggestive of a PIDD, with normal lymphocyte subsets and normal immunoglobulin levels. ${ }^{[9,10]} \mathrm{A}$ high index of clinical suspicion is therefore important. The phenotypic spectrum of MSMD is wide. ${ }^{[1,9,10]}$ In our cohort, the clinical presentation ranged from some patients having earlyonset severe disease to others having persistent, difficult-to-manage infections and two identified through genetic testing but clinically asymptomatic. Unusual presentation or unusual mycobacterial isolates, such as BCG/NTM, were particular flags for MSMD in this cohort, which is in keeping with typically described MSMD presentations. ${ }^{[1,9,10]}$ Infection with MTBC predominated, in contrast to the developed-world setting, ${ }^{[10,16]}$ suggesting that in a hyperendemic TB setting, MTBC are the predominant infecting organisms in MSMD.

In patients with suspected MSMD who had PBMCs available, functional immunophenotyping showed an impaired IL-12-IFN- $\gamma$ pathway, giving an overview of the functionality of this pathway. It facilitated functional confirmation of pathogenic variants and proved particularly useful in patients with a VUS. Although the VUSs require further functional phenotyping to confirm a pathogenic variant, the impaired IL-12-IFN- $\gamma$ pathway with SPUR infection was considered highly suggestive of MSMD. Such cases were identified as requiring heightened surveillance and long-term follow-up, facilitating earlier diagnosis of mycobacterial or other significant infection. In combination with clinical assessment, immunophenotyping can therefore be an important tool in interpreting molecular data as well as deciding on whether to offer IFN- $\gamma$ supplementation and recall for suspected dissemination. 


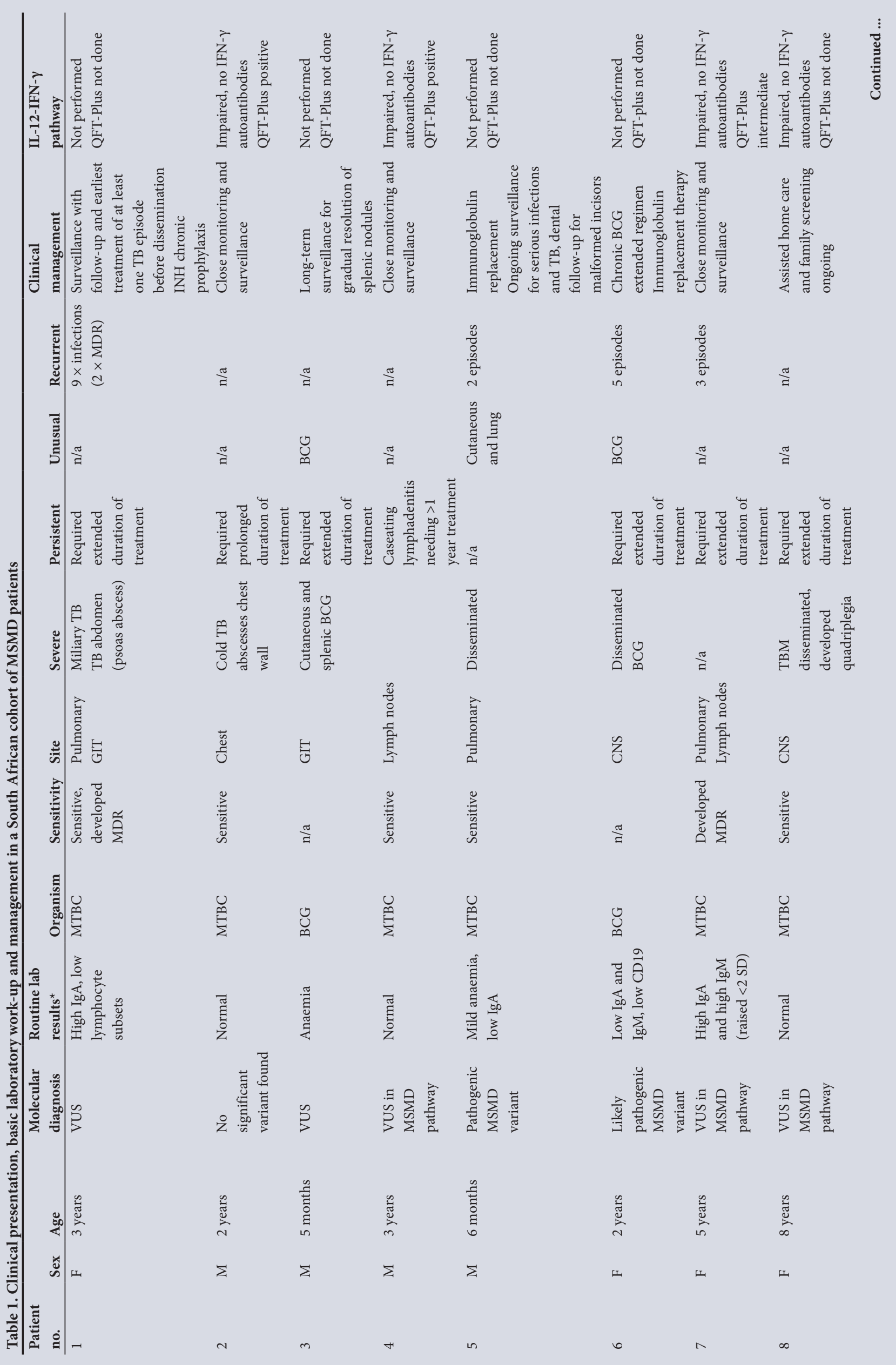




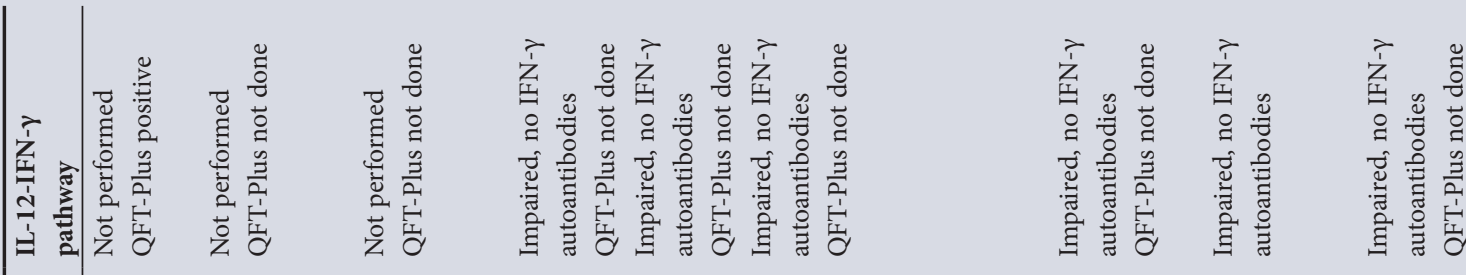

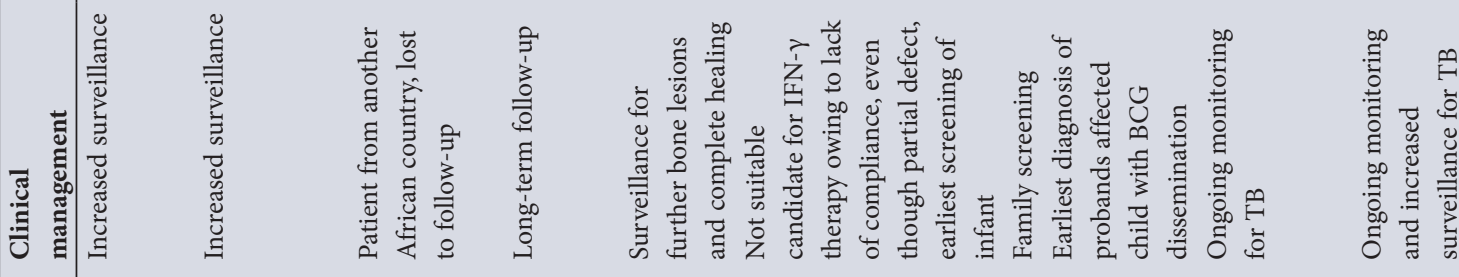

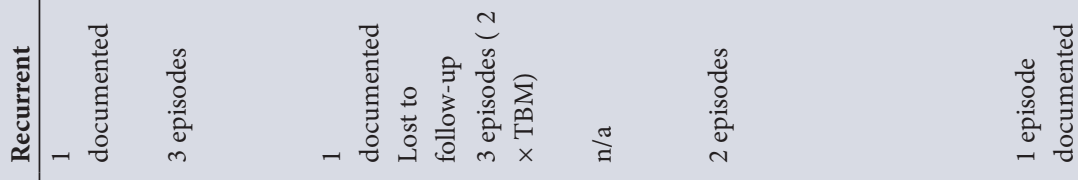

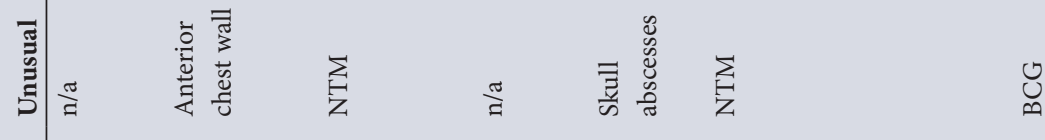

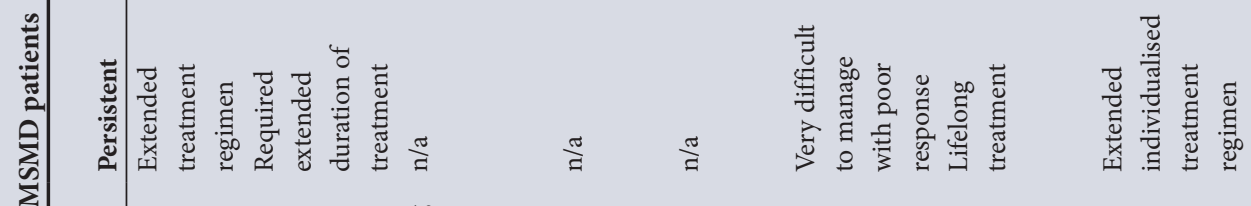

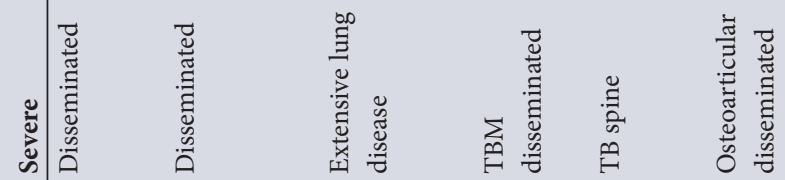

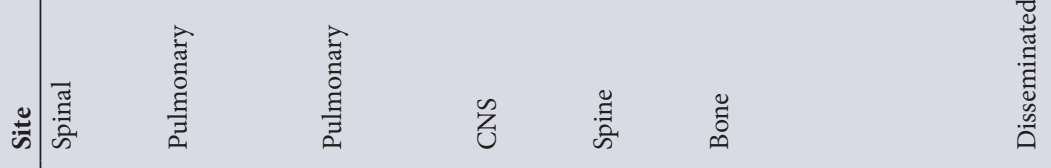

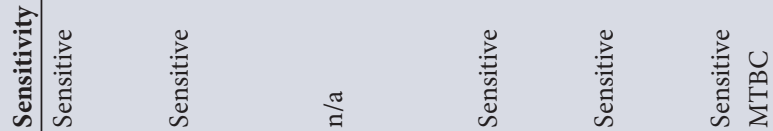

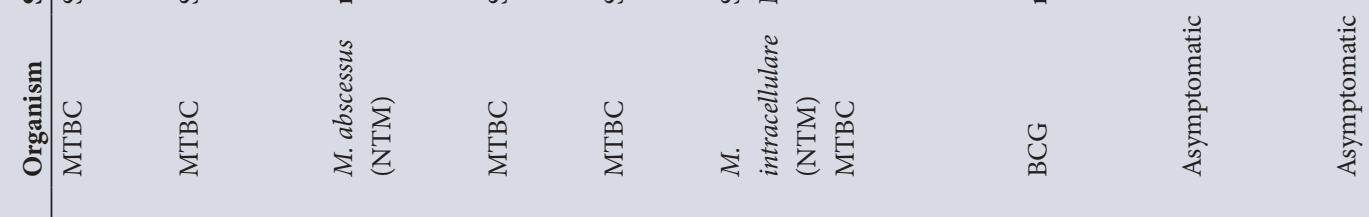

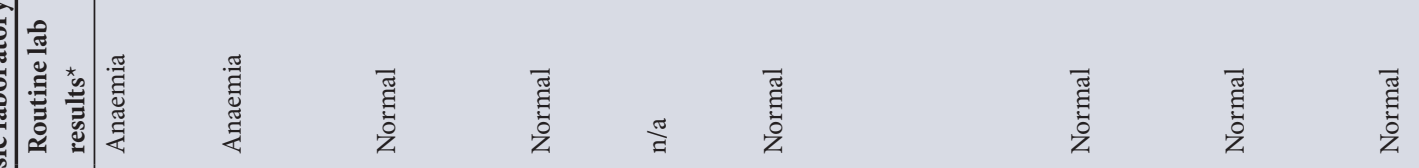

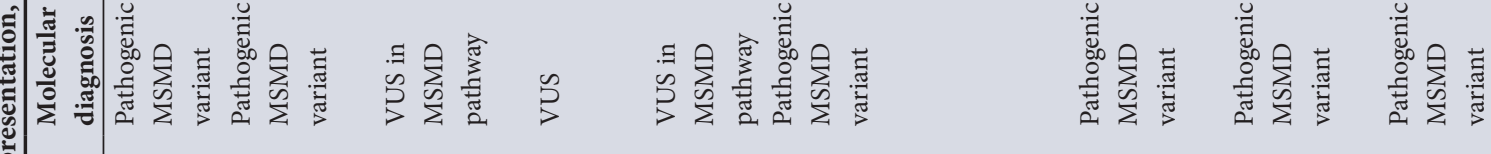
:

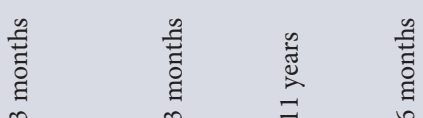

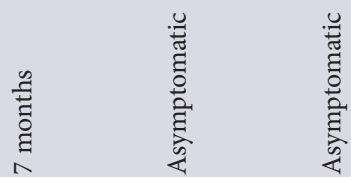

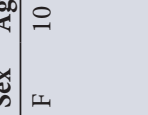

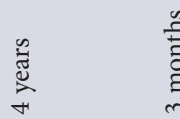
घू
世

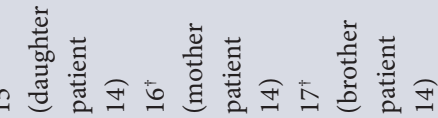




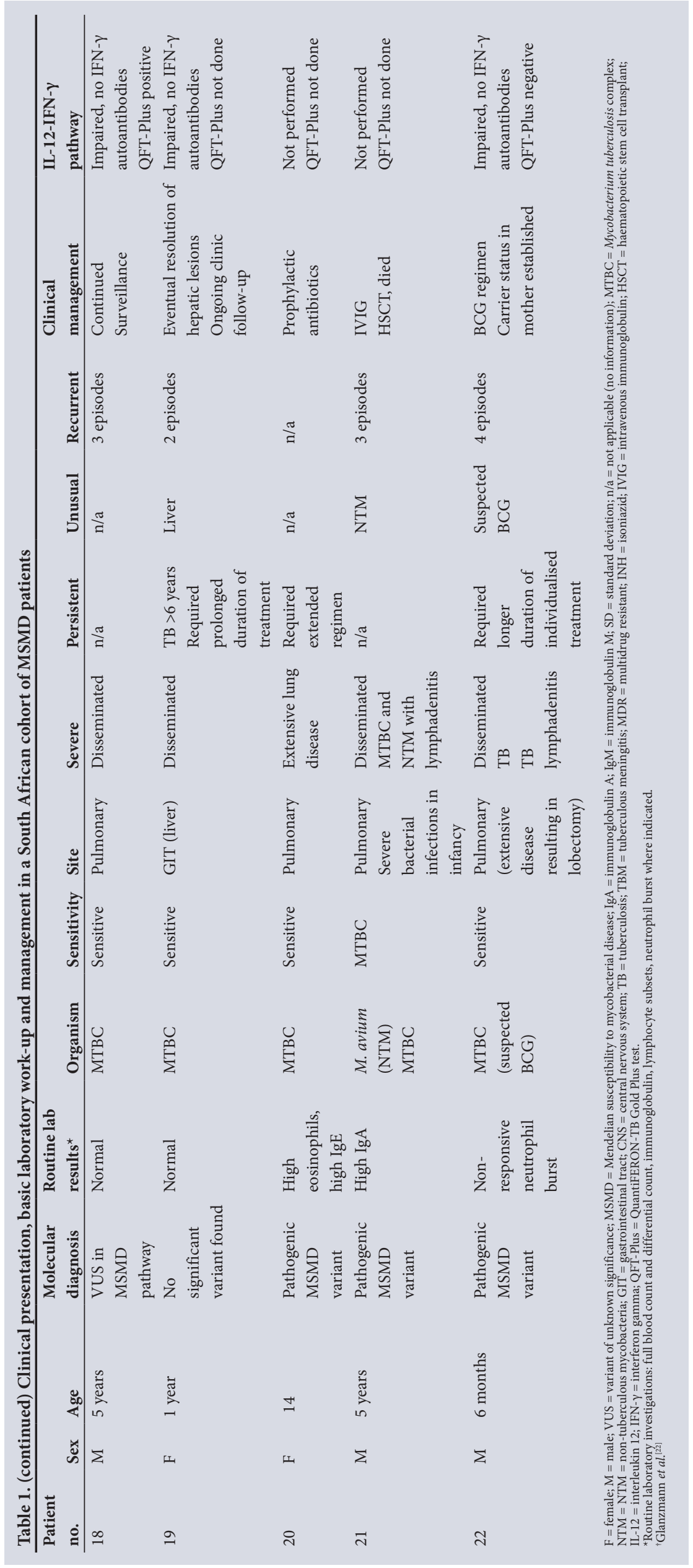

Laboratory diagnosis of BCG disease remains a challenge, emphasising the importance of clinical suspicion and close laboratory collaboration in such cases. A negative QTF-Plus may be useful in this setting, as is highlighted by patient 22, whose QTF-Plus was negative despite cultured MTBC. With the clinical suspicion of BCG dissemination and impaired IL-12IFN- $\gamma$ response on immune assays as well as an impaired neutrophil burst response and molecular diagnosis confirming chronic granulomatous disease, the patient was managed for BCG infection and responded well.

Genomic testing is becoming the gold standard for diagnosis in PIDD. ${ }^{[1,2,7]}$ The application of WES in this cohort of patients confirmed a molecular diagnosis in just over one-third of patients, in keeping with international and local identification of a molecular diagnosis with the application of NGS, $40 \%$ and $30 \%$, respectively. ${ }^{[17,18]}$ Sensitivity for NGS in undiagnosed PIDD has been reported to range from $83 \%$ to $100 \%$, while specificity ranges from $45 \%$ to $99 \%{ }^{[2,17]}$ The diagnostic yield depends largely on the population as well as the technical skill with which and the circumstances under which NGS is employed. ${ }^{[2,17,18]}$ Using WES and whole-genome sequencing (WGS) to diagnose rare and understudied diseases remains a challenge that is further complicated when done in a population with a poorly defined reference genome, such as South Africans. ${ }^{[7,13,18]}$ The large amounts of data generated by WGS and WES that require highly skilled interpretation complicate their use in routine clinical practice, so clinically guided PIDD panels are generally preferred in the initial diagnostic work-up. ${ }^{[2,18]}$ An MSMD-targeted gene panel should focus on mutations in the IFNGR1, IFNGR2, STAT1, CYBB, IRF8, NEMO, IL12B, IL12RB1 and IL12RB2 genes. ${ }^{[1,9,10]}$ In SA, WES and WGS are generally only available on a research basis owing to limited funding for genomic medicine. This said, patients at Tygerberg Hospital do have access to state-funded gene panels. Bioinformaticians, clinical and molecular geneticists/genetic counsellors and clinical immunologists are key in variant interpretation and deciding whether variants are pathogenic or likely to be pathogenic and therefore carrying the validity that is needed in deciding on a definitive treatment regimen, carrier tracing and prognosis.

The seemingly low detection rates reflected both internationally and locally illustrate the complexity and heterogeneity of PIDD. ${ }^{[2,7,19,20]}$ Although the accuracy of SPUR definitions for MSMD is low, it is a non-invasive, cheap bedside approach and its true performance can only be assessed 
after it is put into use with molecular screening. In cases with a confirmed molecular diagnosis, treatment regimens were altered by increasing clinical surveillance, using drug-resistant regimen combinations, extending duration of treatment, giving prophylaxis and discussing supplementation with IFN- $\gamma$.

Owing to the burden of TB in SA, teasing out patients with MSMD is difficult. Many patients are probably missed and their infections presumed to be due to an environment of hyperexposure, socioeconomic circumstances and poor compliance. ${ }^{[13]}$ The present study highlights that even in such contexts, patients do exist and awareness of MSMD and appropriate suspicion are needed on the part of healthcare professionals to ensure that patients are receiving appropriate care. The use of an algorithm may facilitate identification of potential MSMD patients, both clinically and in the laboratory.

Recommendations put forward to clinicians and laboratory personnel to improve the diagnostic yield of patients with underlying MSMD include (Fig. 1):

- In the microbiology laboratory, positive cultures for BCG or NTM in the absence of HIV or another secondary cause for immunodeficiency should be brought to the attention of clinicians to investigate further for the possibility of PIDD.

- In the context of SPUR TB, HIV status must be confirmed, and other causes of secondary immunodeficiency or underlying anatomical pathology (e.g. lung disease) must be excluded by in-depth investigation.

- A tiered investigation approach to PIDD has been proposed and is widely applied: ${ }^{[8,21]}$

- First-line investigation. An FBC and differential count will highlight any cytopenias that may suggest an alternative PIDD diagnosis or other underlying pathology. The CD4 count is widely available and may serve as a screening test in the primary healthcare setting for low T-lymphocytes in the absence of HIV or other secondary immunodeficiency.

- Second-line testing. Immunoglobulin and lymphocyte subsets assist in excluding other PIDD. The QFT-Plus, although unvalidated, could be used as a screening test of the functional IFN- $\gamma$ pathway.

- In SPUR TB, when secondary immunodeficiency or anatomical pathology is absent and other PIDD has been excluded, thirdand fourth-line investigations are then recommended in close consultation with the immunology service.

- Focused immunophenotyping can be done to assess pathway function (usually in a research setting).

- Genetic testing should ideally be done in the context of genetic counselling. It should include either targeted gene panels (focusing on mutations in the IFNGR1, IFNGR2, STAT1, CYBB, IRF8, NEMO, IL12B, IL12RB1 and IL12RB2 genes) or preferably more expanded NGS technologies such as diagnostic-based WES or WGS.

\section{Study limitations}

The PIDD registry does not represent all PIDD patients in SA owing to presumed under-reporting. The SPUR TB definitions used, although guided by expert opinion, have not been validated in MSMD, and further study is needed. Owing to the nature of the study and the rarity of the disease there is selection bias, and this algorithm needs to be applied to the paediatric population as a whole to assess the true sensitivity and specificity; however, this also highlights the importance of publishing these data. Although a small cohort of patients is a limitation in this rare disease, it is still considered important and relevant.

\section{Conclusions}

The SA context of hyperendemic TB provides both an ideal and a challenging setting to review MSMD. SPUR TB infections in otherwise healthy children should be investigated further for possible PIDD, in particular MSMD. In such patient populations, the results of baseline immunological investigations are usually normal, and a PIDD could be missed without focused immunophenotyping and molecular diagnosis. The molecular diagnosis is pivotal to the diagnosis, as it not only guides classification and diagnosis but also influences clinical approach and management. With the dawn of WES and WGS, variant interpretation is complex and needs to be addressed with caution, requiring a multidisciplinary approach and close collaboration between clinical immunologists, bioinformaticians, immunologists, clinical geneticists and genetic counsellors.

\section{Declaration. None.}

Acknowledgements. The authors wish to acknowledge the contribution of all study participants and expert clinicians, Prof. Simon Schaaf and Prof. Helena Rabie, the National Health Laboratory Service (NHLS) Tygerberg Hospital departments of Haematopathology, Medical Microbiology and Immunology, particularly Dr Zivani Chapanduka, Prof. Andrew Whitelaw and technologists Jeremy Goodway, Cornè Rautenbach and Marielize Swartz. They also wish to acknowledge the National Research Foundation (NRF), the South African Medical Research Council (SAMRC) and the Harry Crossley Foundation for their financial support. Research reported in this publication was supported by the SAMRC, the Harry Crossley Foundation, the NRF and the NHLS. The content is solely the responsibility of the authors and does not necessarily represent the official views of the SAMRC.

Author contributions. ME, HMC, RHG, MM and CK conceived the project. BG carried out all DNA sample preparation and carried out bioinformatics analysis of NGS data. Focused immunophenotyping of the IL-12-IFN- $\gamma$ pathway was carried out by AvC. CE assisted with the genetic counselling. $\mathrm{ME}, \mathrm{HMC}$ and DRA were involved in patient recruitment and management. KR provided expert advice and assisted in data analysis and interpretation. All authors read and approved the final manuscript.

Funding. Crick African Network, which receives its funding from the UK's Global Challenges Research Fund (MR/P028071/1), NHLS (GRANT004 94525), NRF Research and Development Fund (unique grant number 93360), Department of Science and Innovation-NRF Centre of Excellence in Biomedical Research (UID 41744), SAMRC Centre for Tuberculosis Research.

Conflicts of interest. None.

1. Bousfiha A, Jeddane L, Picard C, et al. Human Inborn Errors of Immunity: 2019 Update of the IUIS Phenotypical Classification. J Clin Immunol 2020;40(1):66-81. https://doi.org/10.1007/s10875-02000758-x

2. Seidel MG, Kindle G, Gathmann B, et al. The European Society for Immunodeficiencies (ESID) Registry Working Definitions for the Clinical Diagnosis of Inborn Errors of Immunity. J Allergy Clin Immunol Working Definitions for the Clinical Diagnosis of Inborn Errors of I
Pract 2019;7(6):1663-1770. https://doi.org/10.1016/j.jaip.2019.02.004

Pract 2019;7(6):1663-1770. https://doi.org/10.1016/j.jaip.2019.02.004
3. Seidel M. Autoimmune and other cytopenias in primary immunodeficiencies: Pathomechanisms, 3. Seidel M. Autoimmune and other cytopenias in primary immunodeficiencies: Pathomechanisms,
novel differential diagnoses, and treatment. Blood 2014;124(15):2337-2344. https://doi.org/10.1182/ blood-2014-06-583260

4. Casanova JL, Abel L. Human genetics of infectious diseases: A unified theory. EMBO J 2007;26(4):915922. https://doi.org/10.1038/sj.emboj.7601558

5. Whittaker E, López-Varela E, Broderick C, Seddon JA. Examining the complex relationship between tuberculosis and other infectious diseases in children. Front Pediatr 2019;7:233. https://doi.org/10.3389/ fped.2019.00233

6. Seddon JA, Chiang SS, Esmail H, Coussens AK. The wonder years: What can primary school children teach us about immunity to Mycobacterium tuberculosis? Front Immunol 2018;9:2946. https://doi. org/10.3389/fimmu.2018.02946

7. Glanzmann B, de Villiers N, Möller M, et al. A primary immunodeficiency diseases odyssey - answer from the exome in a case with immunodeficiency and autoimmunity. Curr Allergy Clin Immuno 2017;30(4):278-283. http://www.embase.com/search/results?subaction=viewrecord\&from=export\&id $=$ L620681203 (accessed 7 February 2021).

8. Eley B, Esser M. Investigation and management of primary immunodeficiency in South African children. S Afr Med J 2014;104(11):1-4. https://doi.org/10.7196/SAMJ.8946 
9. Deyà-Martínez À, Juan M, Martin-Nalda A, et al. Laboratory evaluation of the IFN- $\gamma$ circuit for the molecular diagnosis of Mendelian susceptibility to mycobacterial disease. Crit Rev Clin Lab Sci 2018;55(3):184-204. https://doi.org/10.1080/10408363.2018.1444580

10. Rosain J, Kong XF, Martinez-Barricarte R, et al. Mendelian susceptibility to mycobacterial disease Rosain J, Kong XF, Martinez-Barricarte R, et al. Mendelian susceptibility to mycobacterial dis
2014 - 2018 update. Immunol Cell Biol 2019;97(4):360-367. https://doi.org/10.1111/imcb.12210

11. Kaushansky K, Prchal JT, Press OW, Litchman MA, Levi M, Burns LJCM. Williams Haematology. 9th ed. New York: McGrawHill, 2016.

12. Bustamante J, Boisson-Dupuis S, Abel L, Casanova JL. Mendelian susceptibility to mycobacterial disease: Genetic, immunological, and clinical features of inborn errors of IFN- $\gamma$ immunity. Semin Immunol 2014;26(6):454-470. https://doi.org/10.1016/j.smim.2014.09.008

13. Glanzmann B, Uren C, de Villiers N, et al. Primary immunodeficiency diseases in a tuberculosis endemic region: Challenges and opportunities. Genes Immun 2018;20:447-454. https://doi. org/10.1038/s41435-018-0041-0

14. Lee WI, Huang JL, Yeh KW, et al. Immune defects in active mycobacterial diseases in patients with primary immunodeficiency diseases (PIDs). J Formos Med Assoc 2011:110(12):750-758. https://doi org/10.1016/j.jfma.2011.11.004

15. Wiseman CA, Gie RP, Starke JR, et al. A proposed comprehensive classification of tuberculosis disease severity in children. Pediatr Infect Dis J 2012.31(4):347-352. https://doiorg/10.1097/ INF.0b013e318243e27b

16. Casanova J-L. Human genetic basis of interindividual variability in the course of infection. Proc Natl Acad Sci 2015;112(51):E7118-E7127. https://doi.org/10.1073/pnas.1521644112
17. Yska HAF, Elsink K, Kuijpers TW, Frederix GWJ, van Gijn ME, van Montfrans JM. Diagnostic yield of next generation sequencing in genetically undiagnosed patients with primary immunodeficiencies: of next generation sequencing in genetically undiagnosed patients with primary immunodeficiencies: A syster

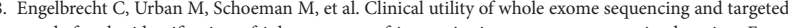
panels for the identification of inborn errors of immunity in a resource-constrained setting. Front Immunol 2021;12:665621. https://doi.org/10.3389/fimmu.2021.66562

19. Arts P, Simons A, Alzahrani MS, et al. Exome sequencing in routine diagnostics: A generic test for 254 patients with primary immunodeficiencies. Genome Med 2019;11(1):1-15. https://doi.org/10.1186/ s13073-019-0649-3

20. Bisgin A, Boga I, Yilmaz M, Bingol G, Altintas D. The utility of next-generation sequencing for primary immunodeficiency disorders: Experience from a clinical diagnostic laboratory. Biomed Res Int 2018;9647253. https://doi.org/10.1155/2018/9647253

21. Erjaee A, Bagherpour M, van Rooyen C, et al. Primary immunodeficiency in Africa - a review. S Afr Med J 2019;109(8):3-11. https://doi.org/10.7196/SAMJ.2019.v109i8b.13820

22. Glanzmann B, Möller M, Moncada-Velez M, et al. Autosomal dominant IFN- $\gamma$ R1 deficiency presenting with both atypical mycobacteriosis and tuberculosis in a BCG-vaccinated South African patient. J Clin Immunol 2018;38(4):460-463. https://doi.org/10.1007/s10875-018-0509-8

Accepted 31 May 2021. 